\title{
Estudo evolutivo da anatomia das artérias coronárias em espécies de vertebrados com técnica de moldagem em acetato de vinil (vinilite)
}

\author{
Tânia Maria de Andrade RODRIGUES*, José Arnaldo O. PALMEIRA*, \\ José Teles de MENDONÇA*, Otoni Moreira GOMES**
}

RBCCV 44205-475

\begin{abstract}
Rodrigues T M A, Palmeira J A O, Mendonça J T, Gomes O M - Estudo evolutivo da anatomia das artérias coronárias em espécies de vertebrados com técnica de moldagem em acetato de vinil (vinilite). Rev Bras Cir Cardiovasc 1999; 14 (4): 331-9.
\end{abstract}

RESUMO: No presente trabalho foram produzidos 30 moldes anatômicos de corações de vertebrados, visando contribuir para o estudo das artérias coronárias direita e esquerda de diferentes espécies: peixes, anfíbios, répteis, aves e mamíferos. Os corações foram injetados com acetato de vinil, submetidos a corrosão e semicorrosão pelo ácido clorídrico, a fim de evidenciar o padrão anatômico apresentado pelas artérias coronárias no tocante à evolução das espécies e adaptações morfológicas (estrutura e arquitetura). Com base na morfologia das peças estudadas foram obtidas as seguintes conclusões: a técnica utilizando acetato de vinil, associada à corrosão, mostrou-se eficaz na produção de modelos de coração de diferentes espécies, apresentando detalhamento capaz de permitir visibilização dos ramos colaterais, quando existentes; o número de estruturas e a complexidade vascular cardíaca aumenta na medida em que os seres evoluem na escala zoológica. No réptiliguana iguana foi encontrado ventrículo duplo com tríplice via de saída, como único padrão evolutivo da anatomia dos ventrículos e grandes vasos da base ainda não descrito como cardiopatia congênita em humanos.

DESCRITORES: Vasos coronários, anatomia. Vertebrados, anatomia. Modelos anatômicos.

\section{INTRODUÇÃO}

JATENE et al. (1), estudando as artérias coronárias humanas, relatam que as primeiras descrições sobre elas datam de 1706 feitas por Raymond Vieussens.

De aquisição relativamente recente, as angiotécnicas para estudo da vascularização cardíaca têm sido de grande valia para compreensão da morfologia das artérias coronárias, seja utilizando injeção de substâncias corantes, radiopacas e mercuriais, seja por diafanização ou pela corrosão.

PIANETTO \& ARGENTINA (2), em 1939, empregaram método com remoção do coração imediatamente após a morte, colocando-o em refrigerador entre $5^{\circ} \mathrm{C}$ e $10^{\circ} \mathrm{C}$, sendo o sistema coronário lavado com água destilada e mistura colorida radiopaca injetada, para, em seguida, serem feitos roentgeno-

Trabalho realizado no Departamento de Morfologia do Centro de Ciências Biológicas e da Saúde da Universidade Federal de Sergipe e na Fundação Cardiovascular São Francisco de Assis - ServCor - Belo Horizonte, MG, Brasil.

Apresentado ao Congresso Nacional de Cirurgia Cardíaca. Fortaleza, CE, 8 a 10 de abril, 1999.

* Do Departamento de Morfologia do Centro de Ciências Biológicas e da Saúde da Universidade Federal de Sergipe.

** Do Curso de Pós-Graduação em Cardiologia e Cirurgia Cardiovascular e Serviço do Coração HSFA - Fundação Cardiovascular São Francisco de Assis. Endereço para correspondência: Tânia Maria de Andrade Rodrigues. Rua Jacuí, 1191. Concórdia. Belo Horizonte, MG, Brasil. CEP: $31110-050$. Tel: (079) 212 6621. e-mail: servicor@servicor.com.br 
Rodrigues T M A, Palmeira J A O, Mendonça J T, Gomes O M - Estudo evolutivo da anatomia das artérias coronárias em espécies de vertebrados com técnica de moldagem em acetato de vinil (vinilite). Rev Bras Cir Cardiovasc 1999; 14 (4): $331-9$

gramas e dissecções de pequenos ramos, usando as radiografias como guia.

ECKSTEIN (3), em 1954, estudou os vasos coronários usando injeção de sulfato de bário com pressão, tempo e temperatura controlados; os corações eram então abertos pelo método proposto por SCHLESINGER (4) e radiografados.

PAIVA \& ANNUNZIATA ${ }^{(5)}$, em 1962, em corações isolados e reduzidos por esvaziamento e lavagem das cavidades ventriculares e atriais, injetaram mediante compressão manual, sucessivamente, as coronárias direita e esquerda com gelatina corada pelo cinábrio.

HOWE et al. (6) e FEHN et al. (7) faziam infusão de sulfato de bário (micropaque) nas artérias coronárias, obtinham radiografias e as dissecavam de acordo com técnica de LUMB \& HARDY (8).

BORELLI $(9,10)$ estudou a distribuição das artérias do nó sinoatrial em bovinos azebuados, injetando massa de TEICHMANN (11) nas artérias coronárias. Após fixação em formol a $10 \%$, essas artérias eram dissecadas com auxílio de lupa e algumas peças diafanizadas pelo método de SPALTEHOLZ (12).

DE PAULA (13) publicou trabalho enfocando as particularidades observadas em artérias coronárias de corações humanos das raças negra e branca. Em algumas peças foram feitos moldes utilizando acetato de vinil seguido de corrosão. Em seguida, o autor discorre sobre os métodos de apresentação dos leitos vasculares cardíacos: inicialmente, era feita a dissecção simples, depois observados com o auxílio de lupas e microscópios; posteriormente usando raios $X$ e suspensão colorida baritada fina e homogênea (água + gelatina + bário + zarcão), injetados nos leitos vasculares, foram radiografadas as peças, que serviram como guia para orientação do processo de dissecção. Outras técnicas também utilizadas por este mesmo autor foram a injeção de mercúrio misturado com cinábrio, gelatina e água e a técnica de injeção de massa de TEICHMAN (11), seguidas de dissecção em corações diafanizados.

AZEVEDO et al. (14) num estudo comparativo das artérias coronárias, entre suínos e cães, utilizaram o método de injeção de látex sob pressão controlada $(12 \mathrm{cmHg})$ de modo a homogeneizar a moldagem interna até os capilares com corrosão subseqüente da matéria orgânica.

A atual evolução da engenharia genética e a possibilidade de interferência com padrões anatômicos de organismos em desenvolvimento reavivam a importância do estudo dos padrões filogenéticos cardiovasculares. A dificuldade na obtenção de informações pormenorizadas sobre detalhes da técnica de estudo com moldes vasculares aplicáveis às diferentes dimensões e texturas observadas na escala animal motivou a presente investigação, que tem como objetivo a sistematização da técnica de moldagem com acetato de vinil (vinilite) para aplicação em animais vertebrados de diferentes espécies como contribuição ao estudo evolutivo da anatomia das artérias coronárias.

\section{MATERIAL E MÉTODOS}

Os ensaios foram conduzidos no Departamento de Morfologia do Centro de Ciências Biológicas e da Saúde da Universidade Federal de Sergipe. Foram utilizados corações de peixes, anfíbios, répteis, aves e mamíferos, constituindo conjunto de 29 peças, sendo 4 peixes, 4 sapos, 2 iguanas, 3 perus, 1 avestruz, 2 ratos, 2 coelhos, 3 suínos, 3 ovinos, 2 bovinos e 4 humanos.

Foram avaliados corações de peixes (Elagatis bipinnulatus) e perus (Melleagus gallopavo) obtidos no Mercado Municipal Tales Ferraz, Aracajú - SE, como também os sapos (Bufo paracnemis), ratos (Rattus norvegicus) variedade albino Wistar, coeIhos (Oryctolagus cuniculus) e iguanas (Iguana iguana), estes provenientes do Biotério da Universidade Federal de Sergipe. Avaliou-se também corações de avestruz (Struthio camelus), de bovinos (Bos taurus), de ovinos (Ovis aris) e de suínos (Sus scrofa) provenientes de frigoríficos privados. Os corações humanos utilizados neste estudo foram obtidos no Instituto Médico Legal de Sergipe.

Esta pesquisa foi aprovada pelo Conselho de Ética do Departamento de Morfologia da Universidade Federal de Sergipe.

Todos os exemplares foram in natura conservados em gelo, identificados, classificados, numerados e catalogados.

Os órgãos isolados foram lavados, esvaziados os átrios e ventrículos e feita a necessária redução dos componentes anatômicos de que vinham acompanhados. Foram dissecadas as artérias coronárias junto à sua origem, e canulados os óstios nos modelos maiores. Nos modelos menores, tais como peixes, anfíbios, ratos e iguanas, fez-se a injeção na aorta, retrogradamente. Em todos os espécimes foi injetado acetato de vinil (vinilite) corado.

Ao longo do processo de obtenção das peças, foi necessário um período inicial de aprendizado da técnica, experimentação de diferentes corantes. Algumas peças foram perdidas, pois a concentração do ácido clorídrico, a 40\%, como recomendado por PALMEIRA (15), mostrou-se inadequada. Este fato ocasionou destruição de peças cardíacas de espécies pequenas e médias. Chegou-se então às con- 
Rodrigues T M A, Palmeira J A O, Mendonça J T, Gomes O M - Estudo evolutivo da anatomia das artérias coronárias em espécies de vertebrados com técnica de moldagem em acetato de vinil (vinilite). Rev Bras Cir Cardiovasc 1999; 14 (4): $331-9$.

centrações utilizadas para peças pequenas (peixe, sapo, iguana, rato, peru e coelho) à $5 \%$; peças médias (suíno e ovino) à $10 \%$ e peças maiores (avestruz, boi e homem) à $20 \%$.

A lavagem também constituiu dificuldade inicial, devido a perda do detalhamento das ramificações do leito vascular das artérias coronárias.

A moldagem vascular foi realizada de acordo com a técnica utilizada no Laboratório de Anatomia da Faculdade de Medicina Veterinária e Zootecnia da Universidade de São Paulo e descrita por RODRIGUES (16), como segue: 1) Lavagem das peças com água corrente; 2) Aquecimento das peças à $37^{\circ} \mathrm{C}$, em banho-maria; 3) Canulação dos óstios das coronárias ou da aorta retrogradamente, a depender do tamanho da peça; 4) Injeção de água destilada para lavagem dos leitos vasculares; 5) Injeção de acetato de vinil colorido diluído em acetona a 100\%; 6) Retirada da agulha e oclusão do óstio com fio de náilon monofilamentar agulhado; 7) Condução da peça imersa em água destilada ao congelador (freezer) para solidificação do acetato de vinil colorido; 8) Início do processo de semicorrosão e/ou corrosão da peça com ácido clorídrico (HCl); 9) Lavagem das peças com jato fino de água para limpeza; 10) Conclusão do processo corrosivo e colocação das peças para secagem; 11) Identificação e prontuário de cada peça.

$\mathrm{Na}$ moldagem vascular das coronárias foram injetados acetado de vinil, corante e acetona a 100\%, segundo a técnica utilizada por AZEVEDO et al. (14) usando mamíferos domésticos. Para evitar extravasamentos do material, foram laqueadas as extremidades com fio de náilon monifilamentar 000 agulhado.

Em cubas de vidro foi realizado o processo de semicorrosão, colocando-se as peças em contato com o ácido clorídrico $(\mathrm{HCl}$; $d=1,186)$ a $26 \%$, $20 \%, 10 \%$ e $5 \%$, utilizando o critério de tamanho para escolher a concentração. Moldaram-se peças com gaze e camada de algodão. A quantidade de ácido colocada foi suficiente para imersão total da peça.

Após este período, as peças foram lavadas em fino jato de água, deixando somente o molde de acetato de vinil e secas a temperatura ambiente.

\section{Preparação do acetato de vinil}

Inicialmente, foram pesados $13 \mathrm{~g}$ de acetato de vinil em pó e $5 \mathrm{~g}$ de tinta a óleo, marca Águia; em proveta, mediu-se $100 \mathrm{ml}$ de acetona à $100 \%$. Em seguida, homogeneizou-se a mistura em recipiente tipo Erlenmeyer com bastão de vidro. Logo após, o Erlenmeyer, já contendo todos os componentes da mistura, foi mantido em banho-maria à $37^{\circ} \mathrm{C}$ até a injeção da solução nas peças.

Quando não utilizada toda solução de acetato de vinil preparada no dia, vedou-se bem a abertura do Erlenmeyer, guardando-o em lugar refrigerado. Para novo uso reaqueceu-se o vinilite colocando o Erlenmeyer em banho-maria.

\section{Técnica de injeção}

Foram utilizadas três seringas plásticas de $3 \mathrm{ml}$ para peças pequenas, $5 \mathrm{ml}$ para médias e $10 \mathrm{ml}$ para grandes. Em cada recipiente tipo Becker, separadamente, foram mantidas, em temperatura ambiente, água destilada e acetona à $100 \%$. Para peças pequenas foram introduzidas e amarradas agulhas na porção ascendente da aorta; para peças grandes e médias, as agulhas foram introduzidas e amarradas diretamente nos óstios das coronárias. Injetou-se retrogradamente na aorta água destilada, acetona e solução com acetato de vinil, nessa seqüência. $O$ excesso da solução da acetato de vinil que transbordou foi removido.

Retirou-se a agulha e amarrou-se a peça, que foi colocada em recipiente com água e posta para refrigerar por período de meia hora.

\section{Técnica de semicorrosão}

A peça foi envolvida com gaze em recipiente já contendo camada de algodão, ambas embebidas em solução à 5\%, $10 \%$ e $20 \%$ de ácido clorídrico $(\mathrm{HCl})$ para peças pequenas, médias e grandes, respectivamente. Cobriu-se a peça com o restante da gaze do recipiente e colocou-se mais camada de algodão, também embebida em ácido, vedando-se bem.

A peça foi assim mantida por sete dias e após esse período, lavada com jato fino de água até que o acetato de vinil ficasse exposto e que todo o material orgânico fosse retirado. Sendo o tempo insuficiente para total retirada desse material, foram substituídos a gaze, o algodão e o ácido. Procedeuse dessa forma até obtenção do material desejado.

Com a peça pronta, deixou-se secar à temperatura ambiente sobre filtro de papel, a fim de evitar que a mesma ficasse aderida, depois de seca, ao local utilizado. Identificou-se o recipiente com rótulos contendo o nome da peça e a primeira data da semicorrosão. Fez-se relatório a cada dia de troca do ácido onde foram anotados o seu estado, a última data, seja ela da primeira ou da última troca, e a data na qual foi realizada a observação. 
Rodrigues T M A, Palmeira J A O, Mendonça J T, Gomes O M - Estudo evolutivo da anatomia das artérias coronárias em espécies de vertebrados com técnica de moldagem em acetato de vinil (vinilite).

Rev Bras Cir Cardiovasc 1999; 14 (4): 331-9

\section{Técnica para o preparo de acetona pura}

Com a finalidade de purificar a acetona, essencial no correto desenvolvimento da técnica, trabaIhou-se com a destilação fracionada, procedimento capaz de separar dois componentes de uma mistura de líquidos com pontos próximos de ebulição. Sendo a destilação um processo em que as primeiras frações apresentam maior quantidade do componente mais volátil, em comparação à mistura original composta de acetona e álcool (etanol), foi possível obter acetona mais próxima de $100 \%$ de pureza.

Para realizar-se a destilação fracionada foram necessários os seguintes materiais: 1) Condensador; 2) Adaptador para condensador; 3) Frasco de destilação; 4) Cabeça de destilação; 5) Termômetro; 6) Adaptador de termômetro; 7) Suportes; 8) Fragmentos esféricos de vidro ou metal; 9) Manta aquecedora e reostato; 10) Coluna de Fracionamento; 11) Frasco coletor de destilado.

A técnica da destilação fracionada consistiu em, depois de acondicionar a coluna de fracionamento e montar o equipamento, ferver a mistura de acetona, (ponto de ebulição $56,2^{\circ} \mathrm{C}$ ), e álcool (ponto de ebulição $78,3^{\circ} \mathrm{C}$ ). Os vapores e o condensado da mistura, agitado constantemente pelas pequenas esferas de vidro, passaram pela coluna de fracionamento, onde foram condensados e revaporizados continuamente. Cada revaporização do condensado correspondeu a uma destilação simples, obtendo-se um destilado sucessivamente mais rico no componente de ponto de ebulição mais baixo, no caso a acetona. O enriquecimento substancial do vapor da acetona ocorreu quando este alcançou a cabeça da destilação. A partir daí, o vapor entrou no condensador, onde foi resfriado por água e novamente passou para o estado líquido, dirigindo-se finalmente ao frasco coletor. No frasco coletor obteve-se acetona pura, separada do álcool etílico, que continuou no frasco inicial da destilação. Durante todo o processo da destilação fracionada foi essencial a observação do termômetro para o controle da temperatura desejada, regulada pelo termostato. Para obter um melhor resultado, foi preferível coletar considerável número de pequenas frações a grandes amostras, para garantir a ausência de impurezas.

As peças foram fotografadas, para posterior documentação e realização das observações e descrições de acordo com NETTER (17), SISSON \& GROSSMAN (18), BRACEGIRDIE \& MILES (19).

\section{RESULTADOS}

Não houve diferenças nas concentrações do corante, da acetona pura e do acetato de vinil em pó, sendo as mesmas proporções empregadas para o estudo em todas as espécies.

O endurecimento do vinilite, em todos os experimentos conduzidos, foi satisfatório no período de $30 \min$ à $4^{\circ} \mathrm{C}$. Para a corrosão, a concentração do ácido clorídrico variou de $5 \%$ a $20 \%$ e ocorreram diferenças importantes no tempo de corrosão necessário, com variações de 13 a 47 dias.

Com relação à circulação coronária, ocorreram variações importantes entre as espécies, conforme descrito a seguir:

No peixe (Peixe arabaiana, Elagatis bipinnulatus), não foi possível a definição da anatomia da circulação coronária pelo molde vascular com vinilite pois não foram encontrados ramos vasculares emergindo do bulbo aórtico; a anatomia cardíaca das peças geradas revelou a presença de bulbo aórtico, átrio único e ventrículo único (Figura 1). Em anfíbios (Sapo, Bufo paracnemis), a anatomia cardíaca definiu-se pela presença de átrio direito e esquerdo, com septação interatrial completa; identificou-se o seio venoso como unidade separada do átrio direito; o ventrículo mostrou-se único; notou-se bulbo aórtico conectado ao ventrículo único por valva única; não foram encontrados ramos arteriais coronarianos, ou seja, artérias emergindo do bulbo aórtico para nutrir ventrículo e átrio. No réptil (Iguana, Iguana iguana), a anatomia cardíaca caracterizou-se por apresentar átrios direito e esquerdo, com septação completa e

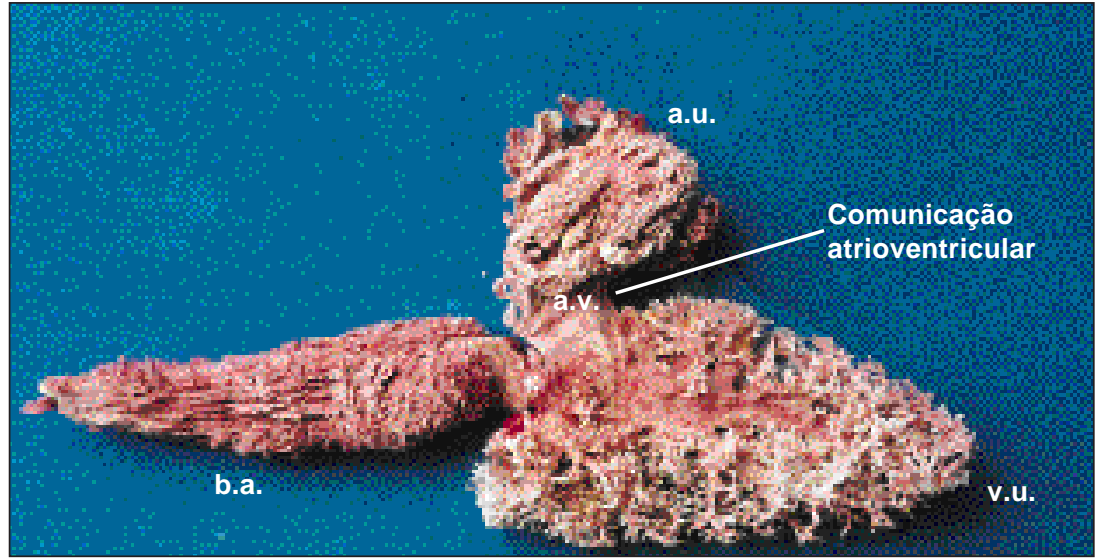

Fig. 1 - Peixe arabaiana (Elagatis bipinnulatus) - Vista ventral do coração, apresentando bulbo arterioso (b.a.) completo, ventrículo único (v.u.), comunicação atrioventricular (a.v.) e átrio único (a.u.) 
Rodrigues T M A, Palmeira J A O, Mendonça J T, Gomes O M - Estudo evolutivo da anatomia das artérias coronárias em espécies de vertebrados com técnica de moldagem em acetato de vinil (vinilite).

Rev Bras Cir Cardiovasc 1999; 14 (4): 331-9.

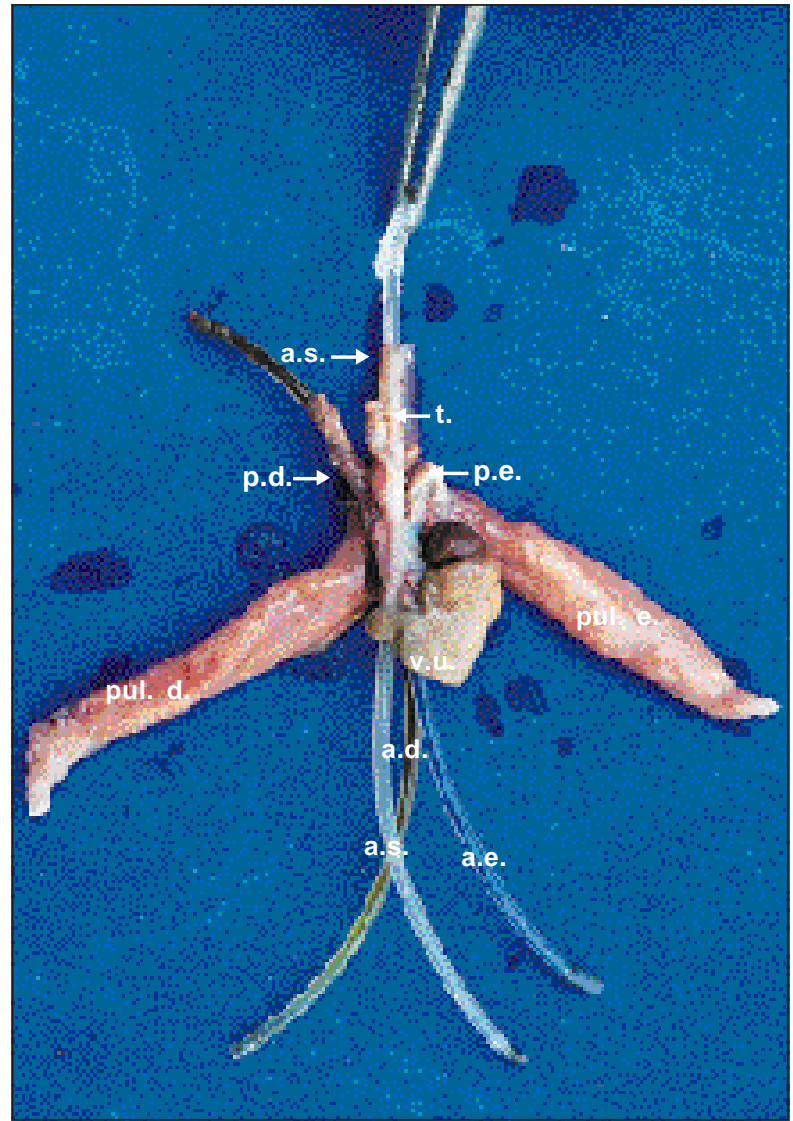

Fig. 2 - Iguana(Iguana iguana) - Vista dorsal, apresentando a tríplice via de saída constituída do arco pulmonar direito (p.d.), arco pulmonar esquerdo (p.e.) e arco sistêmico (a.s.), a partir de ventrículo duplo (v.d.). Notam-se ainda pulmões direito e esquerdo (pul. d..) (pul. e.) e traquéia (t.)

ventrículo duplo, do qual emergiram três vasos: arco sistêmico, arco pulmonar esquerdo e direito (Figuras 2); o sistema coronariano apresentou artéria coronária com tronco único inicial emergindo da aorta para, em seguida, dividir-se em artéria coronária ventral e dorsal (Figura 3). Nas aves (Peru, Melleagus gallopavo), a anatomia cardíaca mostrou duas câmaras atriais e duas ventriculares com septações completas e aorta saindo do ventrículo esquerdo; uma artéria coronária superficial emergiu da aorta por óstio próprio e dirigiu-se para a margem do ventrículo direito; a artéria coronária profunda emergiu do óstio próprio e dirigiu-se também para a margem do ventrículo direito; a artéria coronária profunda emergiu de óstio próprio em tronco único, dirigindo-se para a margem esquerda do ventrículo esquerdo, onde bifurcou-se em artérias circunflexa e artéria interventricular anterior (Figura 4).

Dentre os mamíferos, no rato (Rattus norvegicus) albino da variedade Wistar, a anatomia cardíaca encontrada evidenciou dois átrios, dois ventrículos, valvas atrioventriculares e aorta saindo do ventrículo esquerdo; a anatomia coronariana apresentou artéria coronária direita emergindo de óstio próprio, dirigindo-se para a margem direita do ventrículo direito, com seu ramo interventricular posterior, e a artéria coronária esquerda emergindo de óstio próprio, bifurcando-se, em seguida, em artéria circunflexa e artéria interventricular anterior com ramo diagonal. No coelho (Oryctolagus cuniculus), a anatomia coronariana definiu-se pela emergência da artéria coronária direita de óstio

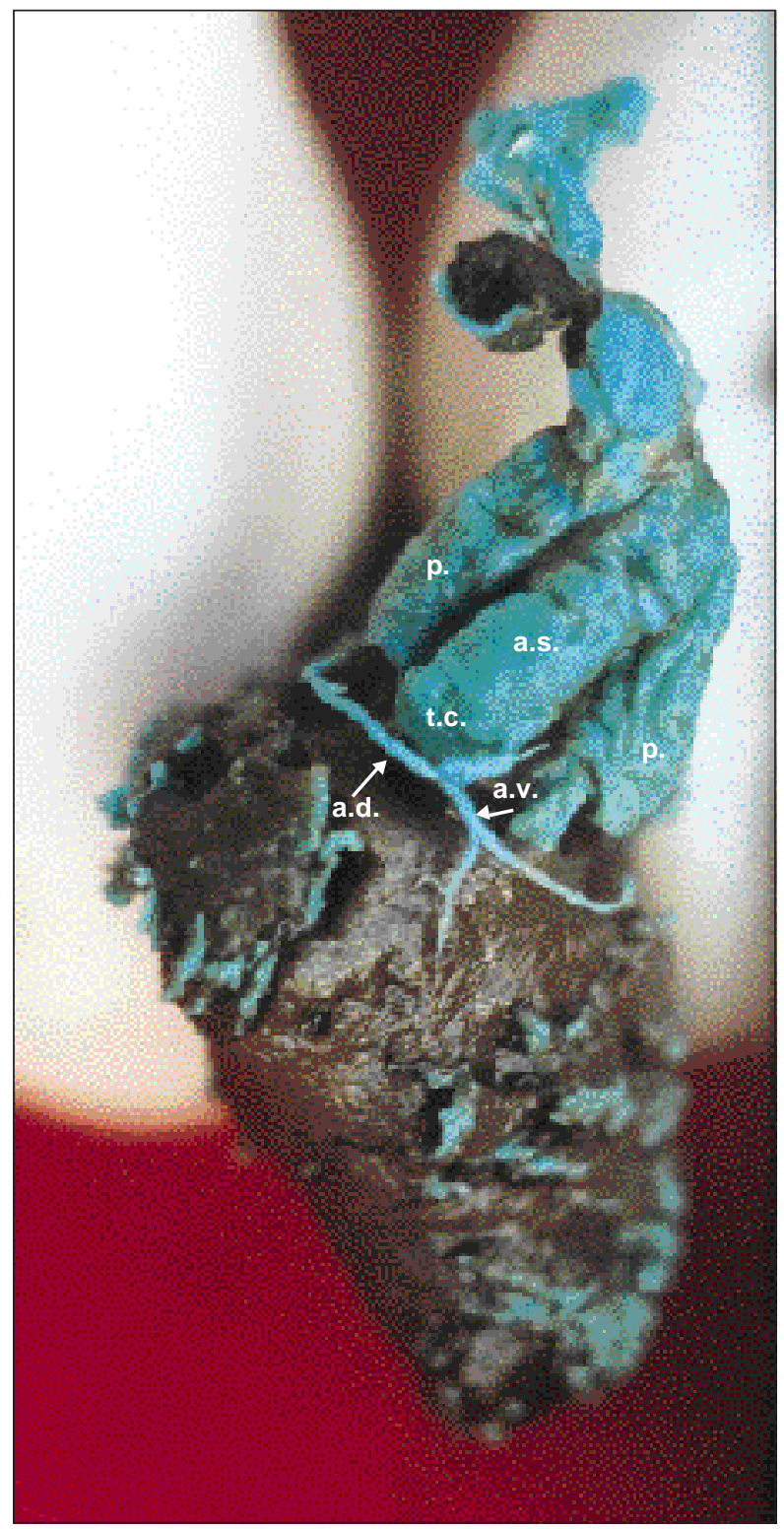

Fig. 3 - Iguana (Iguana iguana) - Vista ventral do coração em proces so de corrosão, mostrando-se o ventrículo duplo, com emergência do arco sistêmico (a.s.) e arcos pulmonares (p.). Nota-se a origem das artérias coronárias ventral (a.v.) e dorsal (a.d.) em tronco comum (t.c.) 
Rodrigues T M A, Palmeira J A O, Mendonça J T, Gomes O M - Estudo evolutivo da anatomia das artérias coronárias em espécies de vertebrados com técnica de moldagem em acetato de vinil (vinilite). Rev Bras Cir Cardiovasc 1999; 14 (4): $331-9$

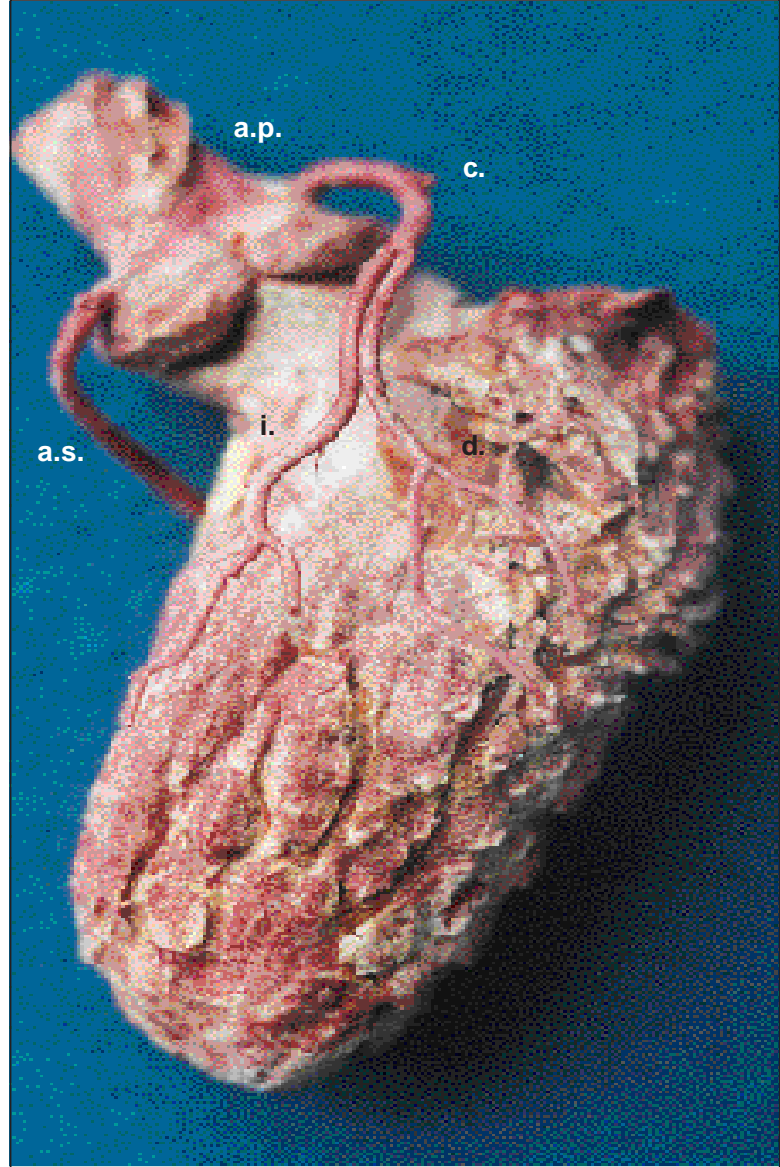

Fig. 4 - Peru(Melleagus gallopavo) - Vista ventral da aorta que se projeta à direita notando-se os seios de Valsalva com origem da artéria coronária superficial (a.s.), dirigindo-se posteriormente e da artéria coronária profunda (a.p.), bifurcando em artéria circunflexa (c.) e artéria interventricular (i) com seu ramo diagonal (d.)

próprio, dirigindo-se para a margem do ventrículo direito originando o ramo interventricular posterior. A artéria coronária esquerda também emergiu de óstio próprio, dirigiu-se para a margem do ventrículo esquerdo, bifurcando-se pouco antes de atingir a margem propriamente dita, em ramo interventricular anterior e ramo circunflexo, que emitiu ramos ventriculares posteriores (Figura 5). Em suíno (Sus scrofa), a anatomia coronariana caracterizou-se pela presença de artéria coronária direita com seu ramo descendente subsinuoso e a artéria coronária esquerda, com seus ramos interventricular paraconal e circunflexo. No ovino (Ovis aris), a anatomia coronariana evidenciou a artéria coronária direita dirigindo-se para a margem do ventrículo direito e ramos terminais ventriculares posteriores; a artéria coronária esquerda bifurcou-se nos ramos: circunflexo, interventricular paraconal e interventricular subsinuoso. Em bovino (Bos taurus), a anatomia cardíaca macroscópica não diferiu da descrita para mamíferos,

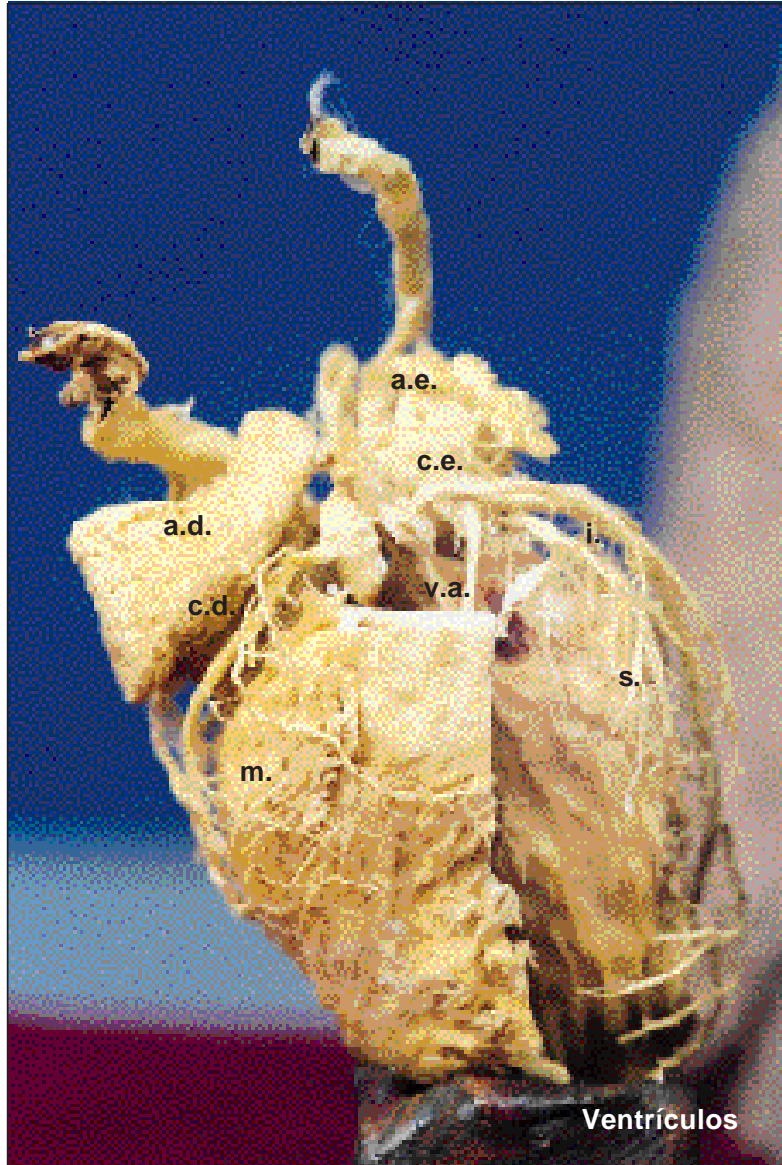

Fig. 5 - Coelho (Oryctolagus cuniculus) - Vista ventral, apresentan do átrios direito (a.d.) e esquerdo (a.e.), emergência da artéria coronária esquerda (c.e.), com seu ramo interventricular anterior (i.) e septais (s.) e a artéria coronária direita (c.d.) com ramo marginal (m.).

exceto pelas dimensões maiores. A anatomia coronariana mostrou a artéria coronária esquerda bifurcando-se nos ramos interventricular paraconal e circunflexo. A artéria coronária direita originou o ramo interventricular sub-sinuoso. Em coração humano, a anatomia cardíaca macroscópica e das artérias coronárias definiu-se pela presença da artéria coronária direita emergindo de óstio próprio, percorrendo o sulco atrioventricular, dando artéria nodal para o átrio direito, a artéria conal para a região do infundíbulo, e, após contornardo a margem direita, dando ramos marginais, atingindo a região da "crux cordis" e bifurcando-se, terminalmente, em ramo ventricular posterior e artéria interventricular posterior com seus ramos septais posteriores. Do óstio esquerdo originou-se o tronco coronariano esquerdo, bifurcando-se em artéria circunflexa - que ocupou o sulco atrioventricular - com ramos marginais e ventriculares posteriores, e na artéria interventricular anterior, que percorreu o sulco interventricular anterior, com ramos diagonais e septais anteriores. 
Rodrigues T M A, Palmeira J A O, Mendonça J T, Gomes O M - Estudo evolutivo da anatomia das artérias coronárias em espécies de vertebrados com técnica de moldagem em acetato de vinil (vinilite). Rev Bras Cir Cardiovasc 1999; 14 (4): $331-9$.

\section{COMENTÁRIOS}

Dada a importância da morfologia cardíaca, inúmeras pesquisas têm sido realizadas nessa área, procurando ampliar o conhecimento sobre o sistema vascular arterial coronariano.

\section{Técnica de moldagem}

A técnica de moldagem anatômica com o vinilite utilizada nesta investigação apresentou resultados satisfatórios como anteriormente referido por vários autores $(2,3,5,9,10,13)$. Entretanto, na fase inicial de condução dos trabalhos, ocorreram perdas de peças possivelmente devido ao uso do ácido clorídrico à 40\%, como preconizado por RODRIGUES (16), tanto para peças menores (peixe, sapo, rato) como para peças médias (suíno, ovino). Tais perdas ocorreram no período de sete dias após o início do processo, levando à sistematização das concentrações para as que se seguem e que se mostraram mais apropriadas para a técnica utilizada: a) ácido clorídrico em concentração de 5\% para corações menores (peixe, sapo, iguana, rato, coelho); b) 10\% para corações médios (suíno e ovino); c) $20 \%$ para corações maiores (avestruz, bovino e humano), com tempo médio de corrosão variando de 13 a 47 dias.

O uso da técnica de semicorrosão apresentou dificuldades de manuseio e a necessidade de lavagens repetidas ao longo do processo de corrosão. Provocou também perda do detalhamento fino das peças, razão porque passou-se a adotar a técnica de corrosão, com lavagem ao final do processo, resultado em produto de melhor qualidade.

O corante utilizado, inicialmente, foi o carmine. No entanto, os moldes obtidos não apresentaram o contraste esperado. Optou-se então, pela tinta a óleo Águia ${ }^{\circledR}$ ou Acrilex ${ }^{\circledR}$ de diferentes cores (azul, verde, vermelho e amarelo). O uso desse material trouxe maior nitidez, destacando mais apropriadamente os componentes anatômicos estudados.

Peixes - MONTAGNA (20), LAGLER et al. (21), e WALKER (22) demonstraram que em peixes pulmonados ou dipnóicos a irrigação do bulbo aórtico e do ventrículo provém das artérias do segundo par de arcos arteriais branquiais eferentes. KULCHITSKI \& ROMENSKI (23) confirmaram esses achados ao atribuírem como precursores das artérias coronárias, as artérias hipobranquiais provenientes do arco aórtico ventral em peixes pulmonados africanos. Entretanto, RAMSAY ${ }^{(24)}$, em 1968, afirmou que os vertebrados inferiores na escala zoológica não possuem vasos sangüíneos, sendo a difusão seu modo de nutrir o coração.

$\mathrm{Na}$ presente investigação estudando o Elagatis bipinnulatus não foi possível identificar artérias coronárias definidas. Os moldes produzidos confirmam os estudos de RAMSAY (24), provavelmente porque a espécie abordada não é classificada entre os dipnóicos.

Anfíbio - Quanto à irrigação do coração de anfíbios (sapos adultos), GRASSÉ (25) relatou que o mesmo é nutrido pelo sangue circulante nas cavidades cardíacas. Esse conceito foi reforçado por MOORE (26) que não demonstrou emergência de artérias coronárias a partir do bulbo aórtico de sapo, mesmo usando cortes microscópicos da referida área. Os trabalhos de MARCUS (27) e SAWAYA (28) referendam este fato. No entanto, ORR (29) e YOUNG (30) relatam a presença de vasos coronários com características histológicas de veia e não de artéria, encontrados em corações de sapos adultos, na região interventricular e sem origem no bulbo aórtico. DUEELLMAN \& TRUEB (31), nesta década, reafirmam que os tecidos cardíacos obtêm nutrientes e oxigênio do sangue que passa através do órgão, citam trabalhos desenvolvidos com veias coronárias encontradas no ventrículo e que drenam para o seio coronário e sugerem que o suprimento da parede do músculo ventricular e do sistema venoso coronariano provém da mistura de sangue oxigenado e desoxigenado do ventrículo. Os modelos produzidos nesse trabalho concordam com as afirmações prévias de que não existem ramos coronários a partir do bulbo aórtico.

Réptil - BELLAIRS \& UNDERWOOD (32) relataram que em cobras com um só pulmão, encontrase dupla via de saída do ventrículo único, porém, em cobras com dois pulmões e lagartos, ocorre tripla via de saída do ventrículo único. Em nosso estudo com Iguana iguana, comprovamos tripla via de saída em ventrículo duplo constituída por arco sistêmico, arco pulmonar direito e arco pulmonar esquerdo. Quanto ao sistema vascular coronariano foi encontrada a emergência de tronco único de artéria coronária da aorta, à direita, bifurcando-se, em seguida em dois ramos: ventral e dorsal, tendo esse último padrão já sido descrito por ORR (29).

Aves - GETTY (33) trabalhando com galinhas descreveu, através de dissecções, artérias superficiais e artérias profundas mergulhadas no miocárdio do lado ventral e direito do septo interventricular. $\mathrm{Na}$ presente investigação utilizando corações de perus, foram confirmados os dados da literatura $(18,25)$ no que concerne ao padrão anatômico de artérias coronárias para aves. No modelo de avestruz foi observado grau maior de ramificações e anastomoses no sistema arterial coronariano. Por tratar-se de ave de grande porte, corredora, pode-se inferir que o aumento da massa muscular cardíaca tenha levado ao aumento das ramificações coronarianas, como observado no crescimento de coronárias de iguana. 
Rodrigues T M A, Palmeira J A O, Mendonça J T, Gomes O M - Estudo evolutivo da anatomia das artérias coronárias em espécies de vertebrados com técnica de moldagem em acetato de vinil (vinilite). Rev Bras Cir Cardiovasc 1999; 14 (4): $331-9$

Como os humanos estão no topo da escala evolutiva, os modelos produzidos serviram como testemunhas em relação aos vertebrados inferiores. Confirmou-se nesta investigação a maior exuberância das ramificações e anastomoses intercoronarianas no coração humano, quando comparado com todos os outros vertebrados estudados.

É importante ressaltar que o estudo filogênico das espécies faz-se necessário para compreendermos o desenvolvimento individual dos vertebrados (34). Concordando com BRUGGEN (35), ao realizar-se a análise do conjunto dos modelos produzidos em vinilite, reafirma-se que no coração humano a ontogenia repete a filogenia dos vertebrados. Tal afirmativa é também reforçada pelos estudos histológicos utilizando técnicas histoquímicas apresentadas por GALLEGO et al. (36) e WARD et al. (37) com estudos moleculares em aves e mamíferos.

DUTRILLAUX (38), ao discutir a evolução das espécies, doenças conhecidas e mutações cromossômicas, afirmou que a maioria dos defeitos congênitos poderiam ser explicados pelo processo evolutivo e aqueles que não encontram explicação nos ancestrais ocorreram em consequência de mutações cromossômicas. Contudo, no presente estudo, buscando a correlação entre as cardiopatias congênitas e a filogenia, não foram encontrados relatos publicados de ocorrência de tripla via de saída de ventrículo em humanos, como a encontrada na Iguana iguana.

Em análise geral, pode-se concluir que a técnica utilizando acetato de vinil, associado à corrosão, mostrou-se eficaz na produção de modelos de coração e artérias coronárias nas diferentes espécies, oferecendo nível de detalhamento capaz de exibir os ramos colaterais, e que o número de estruturas e a complexidade vascular arterial coronariana aumentou progressivamente com a evolução dos seres na escala zoológica e com o aumento da massa ventricular.

RBCCV 44205-475

Rodrigues T M A, Palmeira J A O, Mendonça J T, Gomes O M - Study of evolutive coronary arteries anatomy in vertebrates using vinyl acetate technique. Rev Bras Cir Cardiovasc 1999; 14 (4): 331-9.

ABSTRACT: Thirty anatomic models of vertebrate hearts were produced in order to study the right and left coronary arteries in different species such as fish, amphibians, reptiles, birds and mammals. The hearts were injected with vinyl acetate and then submited to corrosion and/or partial corrosion with hydrochloric acid to obtain the anatomical coronary artery model allowing correlation with the evolution of the species and its morphological adaptations. Based on these models we concluded that: the technique using vinyl acetate associated to corrosion produced models of coronary vessels with enough details to visualize the branches; the number of structures and the vascular complexity increased as the species evolved in the zoological scale. In the reptile Iguana iguana a three outlet double ventricle was found, which has not yet been reported as a human congenital disease in the literature surveied.

DESCRIPTORS: Coronary vessels, anatomy. Vertebrates, anatomy. Models, anatomic.

\section{AGRADECIMENTOS}

À monitora Swanie Perdigão S. Passos, aos bolsistas do CNPq, Lenise A. Franco e Lavínia M. Barreto, e aos acadêmicos-estagiários, Maria da Glória Portugal, Elaine C. Rodrigues, Tiana N. Freire, Liana C. Costa, Juliana A.Sena e Silva, Ângela B. Santos, Priscila C. Bráz, Vanessa T. Nascimento e Sérgio C. Tavares Filho.

\section{REFERÊNCIAS BIBLIOGRÁFICAS}

1 Jatene F B, Aiello V D, Monteiro R - SOCESP Cardiologia. Segundo Volume. São Paulo: Atheneu, 1996: 1173p.

2 Pianetto M B \& Argentina $R$ - The coronary arteries of the dog. Am Heart J 1939; 18: 403-10.
3 Eckstein R W - Coronary interarterial anastomoses in young pigs and mongrel dogs. Cir Res 1954; 2: 460-5.

4 Schlesinger M J - An injection plus dissection study of coronary artery oclusions and anastomoses. Am Heart J 1938; 15: 528

5 Paiva O M \& Annunziata M - Irrigação do sistema 
Rodrigues T M A, Palmeira J A O, Mendonça J T, Gomes O M - Estudo evolutivo da anatomia das artérias coronárias em espécies de vertebrados com técnica de moldagem em acetato de vinil (vinilite). Rev Bras Cir Cardiovasc 1999; 14 (4): $331-9$.

sinuarterial no Sus scrofa domesticus. Folia Clínica et Biologica 1962; 31: 41-4.

6 Howe B B, Fehn P A, Pensinger R R - Comparative anatomical studies of the coronary arteries of canine and porcine heart. I Free ventricular walls. Acta Anat 1968; 71: 13-21.

7 Fehn P A, Howe B B, Pensinger R R - Comparative anatomical studies of the coronary arteries of canine and porcine hearts. II. Interventricular septum. Acta Anat 1968; 71: 223-8.

8 Lumb G D \& Hardy L B - Technique for dissection and perfusion of heart. Arch Pathol 1964; 77: 233-8.

9 Borelli V - Contribuição ao estudo da irrigação arterial do nó sinu-arterial em bovinos azebuados. Rev Fac Med Vet Zootec Univ São Paulo 1969; 8: 59-117.

10 Borelli V - Contribution à l'étude de l'irrigation artérrille du noeud sinusal du coeur du buffle (Bubalus bubalisLinnaeus, 1758). Rev Fac Med Vet Zootec Univ São Paulo 1976; 13: 123-48.

11 Teichmann K L - Kit als injecktionsma sse und die Methoden der Gefoessinjek-tion mit Deurselbe Krakan, aprid Kleins. 1987.

12 Spalteholz W - Das Durchsichsid tigmachen asl biologisohe Arbeitsmethode. Berlim: Handb. Biol. Arbeitsmethodeu, 1924. Abeitsmetode, 1924. Ast. 9.

13 De Paula W - Estudo estatístico sobre irrigação coronária no coração humano em brancos e negros. Folia Clínica et Biologica 1972; 1: 20-42.

14 Azevedo P P, Leal J, Liege S R, Riella A C M, Wouk A F P F, Popp L G - Estudo comparativo das artérias coronárias cordis em Sus scrofa domesticus L., 1758 (Artiodactyla-Suidae) e Canis familiares L. 1758 (Carnivora-Canidae). Arq Biol Tecnol 1980; 23: 71-81.

15 Palmeira J A V - Notas de aula. São Cristóvão; UFS, 1996. (Apostila).

16 Rodrigues H - Técnicas anatômicas. Vitória: Hildegardo Rodrigues, 1998: 222p.

17 Netter F H - Atlas de anatomia humana. Porto Alegre: Artes Médica, 1998: 548p.

18 Sisson S \& Grossman J D - Anatomia dos animais domésticos. Rio de Janeiro: Salvat, 1981: 952p.

19 Bracegirdie B \& Miles P H - An atlas of chordate structur. London: Heinemann Educational Books 1979: 119p.

20 Montagna W - Anatomia comparada. Barcelona: Ediciones Omega, 1967: 379p.

21 Lagler K F, Bardach J E, Miller R R - Ischthyology. New York: John Willey \& Sons, 1962: 544p.
22 Walker W F - Vertebrate dissertion. Philadelphia: WB Saunders 1975: 425p.

23 Kulchitski K J \& Romenski O I - Evolution of blood vessels of the heart wall. Ontogenez 1995; 2: 174-9.

24 Ramsay J A - Introdução à fisiologia animal. São Paulo: EDUSP e Polígona, 1968: 173p.

25 Grassé P P - Traité de zoologie. Paris: Masson Editeurs, 1954: 1145 p.

26 Moore J A - Physiology of the amphibia. New York: Academic Press, 1964: 540p.

27 Marcus H - Zur stammesgeschichte des herzeus morphol. Jahr 1935; 76: 92-103.

28 Sawaya P - Contribuição para o estudo da fisiologia do sistema circulatório do anfíbio Siphonops animalatus (Mikan). Bol Fac Filosof Cienc Letras Univ 1941; 5: 209-33.

29 Orr R T - Vertebrate biology. Philadelphia: W B Saunders, 1971: 544p.

30 Young J Z - The life of vertebrates. London: Oxford University Press, 1978: 820p.

31 Duellman W E \& Trueb L - Amphibia. Baltimore: Johns Hopkins University Press, 1994: 815p.

32 Bellairs A D A \& Underwood G - The origin of snakes. $J$ Anat 1950; 41: 193-237.

33 Getty R - Anatomia dos animais domésticos. Rio de Janeiro: Interamericana, 1981: 1350p.

34 Goshen R, Gonik B, DeGroot N, Hochberg A - Down's syndrome as a model for the decisive role of maternal lineage in human evolution. Med Hypotheses 1995; 44: 116-8.

35 Bruggen W W - Cardiac design in lower vertebrates: what can phylogeny reveal about ontogeny? Experientiae 1988; 44: 919-30

36 Gallego A, Duran A C, De Andrés A V, Navarro P, Muñoz-Chápuli R - Anatomy and development of the sinoatrial valves in the dogfish (Scyliorhinus canicula). Anat Rec 1997; 248: 224-32.

37 Ward S M, Spinner B J, Dube A et al. - Expression of myosin heavy chain transcripts in normal and cardiac mutant Mexican axolotls. Biochem Mol Biol Int 1996; 38: 113-21.

38 Dutrillaux B - Chromosomal evolution in primates: tentative phylogeny from Microcelus murinus (Prosimian) to man. Hum Genet 1979; 48: 251-314. 